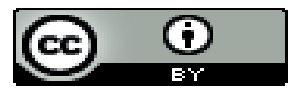

Online

\title{
A study on gender related differences in oxidative stress status
}

\author{
MMR Sardar ${ }^{1 凶}$, MOI Ali $^{2}$, F Pervin $^{3}$, F Yeasmin $^{4}$, N Ahmed ${ }^{5}$, SA Latif ${ }^{6}$
}

\begin{abstract}
Female lives longer than male and incidence of cardiovascular disease is lower in young female. However the role of oxidative stress has been repeatedly described in various diseases but rarely healthy people. The objective of the study was to observe the gender related differences in oxidative stress status and to show significance in alteration. This cross sectional and comparative study was carried out in the Department of Physiology, Rajshahi Medical College during the period from January 2013 to June 2014. Total 45 healthy adult subjects (25 males and 20 females) were enrolled in the study. Systematic sampling technique was used to select each study subject from students, doctors and staffs of Rajshahi Medical College. Malondialdehyde (MDA), a lipid peroxide and alpha-tocopherol, an antioxidant in plasma was measured. MDA:alpha-tocopherol ratio was used as a reliable marker of oxidative stress status. The mean plasma alpha-tocopherol concentration was significantly higher $(p<0.05)$ and plasma MDA: alpha-tocopherol ratio was significantly lower $(p<0.05)$ in female group than male group. The mean plasma alpha-tocopherol concentration was significantly higher $(p<0.05)$ in female group aged 18-21 years than the corresponding male group. From the results of this study, it can be concluded that oxidative stress is less in female than in male indicating presence of potent compensatory response against oxidative stress in female which may be attributed to influence of female sex hormones. Key words: gender, oxidative stress, malondialdehyde, alpha-tocopherol.
\end{abstract}

\section{Introduction}

Oxidative stress results from the imbalance between oxidative and anti-oxidative mechanisms with increased levels of pro-oxidants and depletion of anti-oxidants leading to tissue damage. Oxidative damage to DNA, proteins and lipids induced by the overproduction of reactive oxygen species (ROS) that disrupts cellular homeostasis. On the other hand, the human body generally has anti-oxidant system, which plays an important role in the suppression of ROS over production and protects cells from oxidative stress. Anti-oxidant system is comprised of endogenous compounds (billirubin, uric acid, super oxide dismutases, catalase, glutathione peroxidase, etc) and exogenous compounds (carotenoids, tocopherols, ascorbate, bioflavonoids, etc) ${ }^{1}$

Lipid per-oxidation is an auto-catalytic free radical mediated destructive process

\footnotetext{
1. MMR Sardar, MBBS, MPhil, Assistant Professor of Physiology, Khulna Medical College, Khulna. Email: mijanrmc@hotmail.com

2. MOI Ali, MBBs, MPhil, Associate Professor of Physiology, Rajshahi Medical College, Rajshahi

3. F Pervin, MBBS, MPhil, Assistant Professor of Pharmacology and Therapeutics, Khulna Medical College, Khulna

4. F Yeasmin, MBBS, MPhil, Assistant Professor of Physiology, Diabetic Association Medical College, Faridpur

5. N Ahmed, MBBS, MPhil, Professor of Pharmacology and Therapeutics, Rajshahi Medical College, Rajshahi

6. SA Latif, MBBS, MPhil, Professor of Physiology and Member of Bangladesh Public Service Commission
} 
whereby polyunsaturated fatty acids in cell membranes undergo degradation to form lipid hydro-peroxides. These latter compounds decompose to form a wide variety of products including Malondialdehyde (MDA). ${ }^{2}$ MDA is a three carbon, low molecular weight aldehyde that can be produced from free radical attack on polyunsaturated fatty acids of biological membranes. So, the determination of MDA is used for monitoring lipid peroxidation in biological samples. ${ }^{1}$

Antioxidants are the compounds of exogenous or endogenous in nature which either prevent the generation of toxic oxidants or intercept any that are generated or inactivate them and thereby block the propagation of chain reactions produced by these oxidants. ${ }^{3}$ Tocopherol is a chain breaking antioxidant protecting the lipid phase of the cell from oxidative chain reactions and is an important lipid soluble antioxidant in human plasma. ${ }^{4}$ Alpha-tocopherol is the major form of tocopherol, which is the most potent and present in highest concentration in plasma. So, it exerts the maximum antioxidant effect to prevent lipid peroxidation in plasma. $^{5}$

Female lives longer than male, so it is predicted that the female have lower level of stress. ${ }^{6}$ However, several researchers found no significant difference of MDA levels in male and female population. ${ }^{7,8}$ Moreover, Mendoza-Nunez et al found no significant difference of MDA levels by gender and age group. ${ }^{9}$ On contrary, Kharb and Ghalaut found significantly higher MDA levels in women in comparison to men. ${ }^{10}$ Furthermore, Dittmar et al observed the highest lipid peroxide status in young female group. ${ }^{11}$ On the other hand, Actis-Goretta et al noted higher MDA levels in male. ${ }^{12}$ Additionally, Kasapoglu and Ozben found no gender difference in tocopherol levels. ${ }^{13}$ The results of the studies are not conclusive.

Therefore, the objective of the present study was to examine the biochemical parameters of oxidative stress in healthy human subjects of both genders, to determine the possible gender related differences in oxidative stress status, to compare between antioxidant status (alpha-tocopherol) with pro-oxidant status (MDA).

\section{Materials and Method}

This cross-sectional comparative study was carried out in the Department of Physiology, Rajshahi Medical College during the period from January 2013 to June 2014. Forty five healthy adult subjects of both genders: 25 male and 20 female subjects were enrolled in the study. They were further subdivided into 18-21 years male, 18-21 years female, 41-68 years male and 41-68 years female groups. Study subjects were selected by purposive sampling from medical students, doctors and staffs of Rajshahi Medical College excluding subjects if they had diabetes, hypertension, chronic liver and renal diseases, alcoholism and smoking. The protocol of the study was approved by the Ethical Review Committee of Rajshahi Medical College. Persons taking antioxidant therapy or other drugs, pregnant and lactating women were also excluded from the study.

Before recruitment, aim, benefit and procedure of the study was explained and informed written consent was taken from each study subject. Thorough physical examinations of all subjects were done. Then under aseptic precaution, $4 \mathrm{ml}$ blood in a test tube containing anti-coagulant di-potassium ethylenediaminetetraacetic acid was taken from each subject. Plasma was separated after centrifuging for 15 minutes at $3000 \mathrm{rpm}$. Plasma was used to estimate MDA and alpha-tocopherol levels.

An important point is that we examined only those variables which are most reliable, reproducible and provide a correct assessment of the extent of oxidative stress. Direct measurement of free radicals is difficult due to their unstable and transient nature; therefore, the tendency of free radicals to cause lipid peroxidation was used as an indirect measure. MDA is a three carbon, low molecular weight aldehyde that can be produced from free radical attack on polyunsaturated fatty acids of biological membranes. ${ }^{1}$ So, the determination of MDA was used for monitoring lipid peroxidation. 
We measured alpha-tocopherol as active component of tocopherols because it is a major of lipid soluble chain breaking antioxidant which prevents lipid peroxidation. Moreover, it is the most biologically active form of tocopherols and present in highest concentration. ${ }^{5}$

Plasma was used to estimate MDA levels by the method described by Das et al and Alpha-tocopherol levels were measured by the method described by Baker and Frank. ${ }^{14,15}$

Data were analyzed by a computer using SPSS software program. Statistical analysis was done by Student's $t$-test and a $p$ value $<0.05$ was taken as significant.

\section{Results}

The mean plasma alpha-tocopherol concentration was significantly higher $(p<0.05)$ and plasma MDA:alpha-tocopherol ratio was significantly lower $(p<0.05)$ in female group than male group (Table 1 ).

The mean plasma alpha-tocopherol concentration was significantly higher $(p<0.05)$ in female group aged 18-21 years than the corresponding male group (Table 2).

All the variables were non-significant in female group aged 41-68 years than the corresponding male group (Table 3 ).

\section{Discussion}

In this study, we found that the plasma MDA levels were non-significantly higher in the female subjects than those in the males. This finding is compatible with Kharb and Ghalaut. ${ }^{10}$ It may be due to lack of adequate physical activity or effect of repeated pregnancy during reproductive age in females, which increases oxidative stress. ${ }^{7,16}$

We also found that the plasma MDA levels were non-significantly higher but plasma alpha-tocopherol levels were significantly higher in females than males. Similar trend was observed in aged group by gender. It may be due to compensatory adjustment against oxidative stress in female. ${ }^{6}$ However, in female aged 41-68 years, alpha- tocopherol levels were non-significantly higher than corresponding male group instead of significant rise. It may be due to the fact that with advancing age, female ovarian follicle becomes progressively less sensitive to the effects of gonadotrophins and secrets less sex hormones. ${ }^{17}$ So, effect of sex hormones on anti-oxidant status becomes negligible.

We used plasma MDA:alpha-tocopherol ratio as a marker of oxidative stress status because oxidative stress is determined by imbalance between pro-oxidants and antioxidants. ${ }^{1}$ On the basis of MDA:alphatocopherol ratio, we found that oxidative stress was lower in female than male. It may explain the reason that the female species lives longer than male. ${ }^{6}$ Oxidative stress is more in male than female which may be due to the fact that basal metabolic rate is higher in male than female. ${ }^{18}$ This finding points out that female sex hormones have protective role against oxidative stress status.

In this study, we did not observe any significant difference of oxidative stress status between genders by age groups. These findings are in agreement with MendozaNunez et al. ${ }^{9}$ However, we found that oxidative stress status was non-significantly lower in female group aged 18-21 years as well as 41-68 years in comparison to corresponding male group. It may be due to influence of female sex hormones, which keep the oxidative stress status minimum. ${ }^{19}$ In contrast, Andriollo-Sanchez et al found higher lipid peroxides in females when comparing between middle aged (61 years) and older elderly (71 years)..$^{20}$ So, all of their female subjects were above 60 years and perhaps postmenopausal women. It is possible that their observation reflects the influence of menopause on oxidative stress status. ${ }^{21}$ So, studies are needed to compare the oxidative stress status in between younger premenopausal women and postmenopausal women to find out the contribution of sex hormones on oxidative stress.

In addition, we found that the female group aged 18-21 years had the lowest oxidative stress. It may explain the reason that the 
Table 1. Plasma MDA and alpha-tocopherol in both gender groups, $n=45$

\begin{tabular}{lcc}
\hline Variable & Male $(18-68$ years $)$ & Female (18-68years) \\
& $\mathrm{n}=25$ & $\mathrm{n}=20$ \\
\hline MDA, micro-mol/1 & $3.01 \pm 0.74$ & $3.21 \pm 0.49$ \\
Alpha-tocopherol, mg/dl & $1.74 \pm 0.86$ & $2.57 \pm 0.76^{*}$ \\
MDA:alpha-tocopherol & $2.30 \pm 1.62$ & $1.43 \pm 0.79^{*}$ \\
\hline
\end{tabular}

n, number, ${ }^{*}: p<0.05$ by Student's $t$-test.

Table 2. Plasma MDA and alpha-tocopherol in age matched (18-21 years) gender groups, $n=23$

\begin{tabular}{lcc}
\hline Variable & Male $(18-21$ years $)$ & Female $(18-21$ years $)$ \\
& $\mathrm{n}=10$ & $\mathrm{n}=13$ \\
\hline MDA, micro-mol/1 & $2.49 \pm 0.91$ & $3.03 \pm 0.42$ \\
Alpha-tocopherol, mg/dl & $2.12 \pm 1.04$ & $2.82 \pm 0.50^{*}$ \\
MDA:alpha-tocopherol & $1.52 \pm 1.07$ & $1.09 \pm 0.25$ \\
\hline
\end{tabular}

n, number, ${ }^{*}: p<0.05$ by Student's $t$-test.

Table 3. MDA and alpha-tocopherol in age matched (41-68 years) gender groups, $\mathbf{n}=22$

\begin{tabular}{lcc}
\hline Variable & Male (41-68 years) & Female (41-68 years) \\
& $\mathrm{n}=15$ & $\mathrm{n}=7$ \\
\hline MDA, micro-mol/1 & $3.36 \pm 0.46$ & $3.54 \pm 0.48$ \\
Alpha-tocopherol, mg/dl & $1.48 \pm 0.62$ & $2.11 \pm 0.98$ \\
MDA:alpha-tocopherol & $2.82 \pm 1.74$ & $2.07 \pm 1.07$ \\
\hline
\end{tabular}

n, number.

young females suffer lower incidence of hypertension, ischemic heart disease, atherosclerosis, etc. ${ }^{22}$ On contrary, Dittmar et al observed the highest lipid peroxidation in young females. They used horse reddish peroxidase to measure lipid peroxides. ${ }^{7}$ This finding might be attributed to the interaction between female sex hormones and the test system used for the measurement of lipid peroxides. We assume that the test system for measurement of oxidative stress status might be one of the limitations of their study. So, we suggest that the test results should be compared with those of a second test system.

Additionally, we found that the antioxidant status was higher in the female group aged 18-21 years in comparison to all other groups. It may be due to the fact that the compensatory response of the body against oxidative stress was more potent in younger female. ${ }^{6}$ We assume that female sex hormones, which are highest in young women, are responsible for our obser- vation. ${ }^{17}$ However, Kasapoglu and Ozben stated that tocopherol level does not change with sex. ${ }^{13}$ Their data suggest that various factors may contribute to the process of oxidative stress.

One of the strength of this study is that we measured two closely inter-related biomarkers of oxidative stress status. Consequently the findings indicate that MDA:alpha-tocopherol ratio should be used as marker of oxidative stress status instead of MDA or alpha-tocopherol concentrations alone. However, this was a cross-sectional study. Therefore, it is necessary to carry out a cohort to confirm our findings. We consider a limitation of our study that the study sample was small. Further study using a larger sample size is needed to reach a valid explanation.

\section{Conclusion}

In this study, we found that oxidative stress was less in female than male due to presence of potent compensatory response 
against oxidative stress. We also found that younger female group had the lowest oxidative stress and the highest antioxidant defense. Additionally, we made an attempt to establish the significance of MDA:alphatocopherol ratio as a new marker of oxidative stress rather than concentrations of MDA or alpha-tocopherols alone. However, studies are needed with larger sample size from general population to establish the influence of sex hormones on oxidative stress status.

\section{Conflict of interest: None.}

\section{Acknowledgement}

Authors of this study acknowledge Professor Anwar Habib, Professor of Pharmacology and Therapeutics of Rajshahi Medical College for his support about laboratory work.

\section{References}

1. Suresh DR, Sendil K, Annam V, Hamsaveen A. Age related changes in total antioxidant capacity ratio- a novel marker of oxidative stress. Int J Pharma Bio Sci 2010;1(2):1-6.

2. Knight JA, Pieper RK, McClellan L. Specificity of the thiobarbituric acid reaction: its use in studies of lipid peroxidation. Clin Chem 1988;34(12):2433-8.

3. Mahajan A, Tandon VR. Antioxidants and rheumatoid arthritis. J Indian Rheumatol Assoc 2004;12:139-42.

4. Suhail M, Patil S, Khan S, Siddiqui S. Antioxidant vitamins and lipoperoxidation in non-pregnant, pregnant and gestational diabetic women: erythrocytes osmotic fragility profiles. J Clin Med Res 2010;2(6):266-73.

5. Vatassery GT, Krezowski AM, Eckfeldt $\mathrm{JH}$. Vitamin $\mathrm{E}$ concentrations in human blood plasma and platelets. Am J Clin Nutr 1983;37:1020-4.

6. Vina J, Gamibini J, Lopez-Grueso R, Abdelaziz KM, Jove M, Borras C. Female lives longer than male: role of oxidative stress. Curr Pharm Des 2011;17(36):3959-65.

7. Ozbay B, Dulger H. Lipid peroxidation and antioxidant enzymes in Turkish popula- tion: relation to age, gender, exercise and smoking. Tohoku J Exp Med 2002;197:119-24.

8. Casado A, Castellanos A, LópezFernández ME, Ruiz R, Aroca CG, Noriega F. Relationship between oxidative and occupational stress and aging in nurses of an intensive care unit. Age (Dordor) 2008;30(4):229-36.

9. Mendoza-Núñez VM, Ruiz-Ramos $M$, Sánchez-Rodríguez MA, Retana-Ugalde R, Muñoz-Sánchez JL. Aging-related oxidative stress in healthy human. Tohoku J Exp Med 2007;213:261-8.

10.Kharb S, Ghalaut VS. Plasma lipoperoxides: a preliminary reference range. Indian J Med Sci 2003;57(3):105-7.

11.Dittmar M, Knuth M, Beineke M, Epe B. Role of oxidative DNA damage and antioxidative enzymatic defense systems in human aging. Open Anthropol J 2008;1(1):38-45.

12.Actis-Goretta L, Corrasquedo F, Fraga CG. The regular supplementation with an antioxidant mixture decreases oxidative stress in healthy humans. Gender effect. Clin Chem Acta 2004;349(12):97-103.

13.Kasapoglu M, Ozben T. Alterations of antioxidant enzymes and oxidative stress markers in aging. Exp Gerontol 2001;36(2):209-20.

14.Das BS, Thurnham DI, Patnaik JK, Das DB, Satpathy R, Bose TK. Increased plasma lipid peroxidation in riboflavindeficient, malaria-infected children. Am J Clin Nutr 1990;51(5):859-63.

15.Varley H. Varley's Practical Clinical Biochemistry. 6th ed. London: Heinemann Medical Books; 1988.

16.Amirkhizi F, Siassi F, Minaie S, Djalali M, Rahimi A, Chamari M. Age-related alterations in lipid peroxidation and activities of erythrocyte cytoprotective enzymes in women. Arya Atherosclerosis J 2007;3(2):81-6.

17. Barret KE, Boitano S, Barman SM, Brooks HL. Ganong's Review of Medical Physiology. 24th ed. New York: McGraw-Hill, 2012.

18.18. Khurana I. Essentials of Medical Physiology. New Delhi: Reed Elsevier India Pvt Ltd, 2011. 
19.Abbas AM, Elsamanoudy AZ. Effects of $17 \beta$-estradiol and antioxidant administration on oxidative stress and insulin resistance in ovariectomized rats. Can J Physiol Pharmacol 2011;89(7):497-504.

20.Andriollo-Sanchez M, Hininger-Favier I, Meunier N, et al. Age-related oxidative stress and antioxidant parameters in middle-aged and older European subjects: the ZENITH study. Eur J Clin
Nutr 2005;59(Suppl 2):S58-62.

21.Signorelli SS, Neri S, Sciacchitano S, et al. Behaviour of some indicators of oxidative stress in postmenopausal and fertile women. Maturitas 2006;53(1):77-82.

22. Miller AA, De Silva TM, Jackman KA, Sobey CG. Effect of gender and sex hormones on vascular oxidative stress. Clin Exp Pharmacol Physiol 2007; 34(10):1037-43. 